Pacific Journal of Mathematics

COMPLETE DISTRIBUTIVITY IN LATTICE-ORDERED 


\title{
COMPLETE DISTRIBUTIVITY IN LATTICE-ORDERED GROUPS
}

\author{
RICHARD D. BYRD
}

Throughout this note let $G$ be a lattice-ordered group ("1-group"). $G$ is said to be representable if there exists an 1-isomorphism of $G$ into a cardinal sum of totally ordered groups ("o-groups"). The main result of $\S 3$ establishes five conditions in terms of certain convex 1-subgroups each of which is equivalent to representability. In $\S 4$ it is shown that there is an 1-isomorphism of $G$ onto a subdirect product of 1-groups where each 1-group is a transitive 1-subgroup of all o-permutations of a totally ordered set and that this 1isomorphism preserves all joins and meets if and only if $G$ possesses a collection of closed prime subgroups whose intersection contains no nonzero 1-ideal. Both theorems lead to results concerning complete distributivity.

$G$ is completely distributive if

$$
\bigwedge_{i \in I} \bigvee_{j \in J} g_{i j}=\bigvee_{f \in J} \bigwedge_{i \in I} g_{i f(i)}
$$

where $g_{i j} \in G$ and provided the indicated joins and meets exist. Weinberg [12] has given an equivalent condition to complete distributivity involving arbitrary joins of elements of $G$ (see Proposition 3.5). In [4] Conrad shows that a representable 1-group $G$ is completely distributive if and only if the ideal radical $L(G)$ is zero (ir this paper it was denoted by $R(G)$ ). Using this result we are able to show (Proposition 3.8) that for representable 1-groups the Weinberg condition may be reduced to a condition involving only the joins of pairs of elements. This has been shown by Bernau ([1], Theorem 8) for. Archimedean 1-groups. Holland [7] has shown that each 1-group is 1-isomorphic to a subdirect product of 1-groups $\left\{A_{\lambda} \mid \lambda \in \Lambda\right\}$ where each $A_{\lambda}$ is a transitive 1-subgroup of the 1-group of all o-permutations of a totally ordered set. Theorem 4.6 generalizes the known result for representable 1-groups (see [12] or [4]).

2. Notation and terminology. For the standard definitions and results concerning 1-groups the reader is refered to [2] and [5]. A convex 1-subgroup $M$ of $G$ is called prime if whenever $a$ and $b$ belong to $G^{+}$and not $M$, then $a \wedge b>0$. A convex 1-subgroup (1-ideal) that is maximal with respect to not containing some $g$ in $G$ is called a regular subgroup (regular 1-ideal). Let $\Gamma\left(\Gamma_{1}\right)$ be an index set for the collection $G_{\gamma}\left(I_{\lambda}\right)$ of regular subgroups (regular 1-ideals) of $G$. We 
shall frequently identify these subgroups with their indices. For each $\gamma \in \Gamma\left(\lambda \in \Gamma_{1}\right)$ there exists a unique convex 1-subgroup $G^{\gamma}$ (1-ideal $I^{\lambda}$ ) of $G$ that covers $G_{\gamma}\left(I_{\lambda}\right)$. If $g$ belongs to $G^{\gamma}$ but not $G_{\gamma}$ ( $I^{\lambda}$ but not $I_{\lambda}$ ), then $\gamma(\lambda)$ is said to be a value (ideal value) of $g$. Each regular subgroup is prime. For completeness we state the following theorem, a proof of which may be found in [3].

THEOREM 2.1. For a convex 1-subgroup $M$ of $G$ the following are equivalent.

(1) $M$ is prime.

(2) If $a$ and $b$ belong to $G^{+}$but not $M$, then $a \wedge b$ belongs to $G^{+}$but not $M$.

(3) If $M \supseteqq A \cap B$, where $A$ and $B$ are convex 1-subgroups of $G$, then $M \supseteqq A$ or $M \supseteqq B$.

(4) If $A \supset M$ and $B \supset M$, where $A$ and $B$ are convex 1-subgroups of $G$, then $A \cap B \supset M$.

(5) The lattice $r(M)$ of right cosets of $M$ is totally ordered.

(6) The convex 1-subgroups of $G$ that contain $M$ form a chain.

(7) $M$ is the intersection of a chain of regular subgroups.

If $M$ is normal, then each of the above is equivalent to

(8) $G / M$ is an o-group.

It follows from (6) that the intersection of a chain of prime subgroups of $G$ is prime and hence each prime subgroup exceeds a minimal prime subgroup. If $S$ is a subset of $G$, then [S] will denote the subgroup of $G$ generated by $S$. Again using (6) we state a trivial observation.

CoROLlary 2.2. Let $M_{1}, \cdots, M_{n}$ be convex 1-subgroups of $G$ such that $M_{1}$ is prime. Then $\left[\bigcup_{i=1}^{n} M_{i}\right]=\left[M_{1} \cup M_{k}\right]$ for some $k, 1 \leqq k \leqq n$.

For $0 \neq g$ in $G$ let $R_{g}\left(L_{g}\right)$ be the subgroup of $G$ that is generated by the set of all convex 1-subgroups (1-ideals) not containing $g$. Then $R_{g}\left(L_{g}\right)$ is a convex 1-subgroup (1-ideal) of $G$ and we define the radical and the ideal radical of $G$ respectively to be

$$
\begin{aligned}
& R(G)=\cap R_{g} \quad(0 \neq g \in G) \\
& L(G)=\cap L_{g} \quad(0 \neq g \in G) \text {. }
\end{aligned}
$$

Clearly $L_{g} \subseteq R_{g}$ for all $g$ in $G$ so $L(G) \subseteq R(G)$. A regular subgroup $G_{\gamma}$ (regular 1-ideal $I_{\lambda}$ ) is called an essential subgroup (essential 1-ideal) if there exists $0 \neq h$ in $G$ such that $R_{h} \subseteq G_{\gamma}\left(L_{h} \subseteq I_{\lambda}\right)$. In [4] it was shown that $L(G)$ is the intersection of all essential 1-ideals of $G$ and a similar proof shows that $R(G)$ is the intersection of all essential 
subgroups of $G$. In particular $R(G)$ is an 1-ideal of $G$.

A convex 1-subgroup $C$ of $G$ is said to be closed if whenever $\left\{g_{\alpha} \mid \alpha \in A\right\} \subseteq C$ such that $\bigvee g_{\alpha}$ exists, then $\bigvee g_{\alpha} \in C$. If $a \in G$, then the polar of $a$ is defined to be $p(a)=\{x \in G|| x|\wedge| a \mid=0\} . \quad p(a)$ is a closed subgroup of $G$. If $S \subseteq G$, then we define the polar of $S$ to be $p(S)=\bigcap p(a)(a \in S)$. If $C$ is a convex 1-subgroup of $G$, then $r(C)$ will denote the set of right cosets of $C$ and this set is partially ordered by $C+x \leqq C+y$ if $c+x \leqq y$ for some $c$ in $C$. Then $r(C)$ is a distributive lattice and $C+x \vee C+y=C+x \vee y$ and dually. The empty set will be denoted by $\square, A \backslash B$ denotes the set of elements in $A$ but not in $B$, and $A \subset B$ denotes that $A$ is a proper subset of $B$.

3. Representable 1-groups. Sik [11] proved that an 1-group is representable if and only if all polars are normal. Also in [10] Sik has announced the equivalence of (1) and (4) of Theorem 3.1. The author wishes to thank A. H. Clifford who read a rough draft of this paper and made several valuable suggestions. In particular Clifford noted that in the proof of (1) implies (3), (2) had been proven.

THEOREM 3.1. For an 1-group $G$ the following are equivalent.

(1) $G$ is representable.

(2) If $M$ is a prime subgroup of $G$, then the maximal 1-ideal of $G$ contained in $M$ is prime.

(3) $M$ and $g+M-g$ are comparable for all prime subgroups $M$ of $G$ and for all $g$ in $G$.

(4) Each minimal prime subgroup is normal.

(5) $M$ and $g+M-g$ are comparable for all regular subgroups $M$ of $G$ and for all $g$ in $G$.

(6) Each regular subgroup $M$ of $G$ contains a prime subgroup $N$ such that $N$ is normal in $G$.

Proof. (1) implies (2). Let $M$ be a prime subgroup and let $J$ be the subgroup generated by the collection of all 1-ideals of $G$ that are contained in $M$. Then $J$ is an 1-ideal of $G$. Since $M$ is prime, $p(a) \cong M$ for each $a \in G^{+} \backslash M$. Suppose (by way of contradiction) that $J$ is not prime. Then there exists $b, c$ in $G^{+} \backslash J$ such that $b \wedge c=0$. Therefore $b, c \in M$. Choose $0<a \in G \backslash M . \quad b \notin J$ implies $a \wedge b>0$ and $c \in p(a \wedge b)$ implies $p(a \wedge b) \backslash J \neq \square$. Since $J$ is maximal in $M$, there exists $0<z \in p(a \wedge b) \backslash M$ and since $M$ is prime, $a \wedge z \in G^{+} \backslash M$. But then $b \in p(a \wedge z) \subseteq J$, a contradiction. Therefore $J$ is a prime subgroup of $G$.

(2) implies (3). Let $M$ be a prime subgroup of $G$ and let $g \in G$. By (2) the maximal 1-ideal $J$ of $G$ contained in $M$ is prime. Therefore $J=g+J-g \subseteq g+M-g$. By (6) of Theorem 2.1 it follows that 
$M$ and $g+M-g$ are comparable.

(3) implies (4) since inner automorphisms of $G$ preserve minimal primes.

(4) implies (5). Let $M$ be a regular subgroup and let $N$ be a minimal prime subgroup such that $N \subseteq M$. Then $N=g+N-g \subseteq$ $g+M-g$. Thus $g+M-g$ and $M$ are comparable by (6) of Theorem 2.1.

(5) implies (6). Let $M$ be a regular subgroup of $G$. By (5) $N=\bigcap\{g+M-g \mid g \in G\}$ is the intersection of a chain of regular subgroups, hence by Theorem 2.1 is prime. Clearly $N$ is normal in $G$ and is contained in $M$.

(6) implies (1). For each $0 \neq a \in G$, let $M_{a}$ be a value of $a$. By (6) there exists a prime 1-ideal $N_{a}$ such that $N_{a} \subseteq M_{a}$. By (8) of Theorem 2.1, G/N $N_{a}$ is an o-group. The mapping $x \rightarrow\left(\cdots, N_{a}+x, \cdots\right)$ is an 1-isomorphism of $G$ into the cardinal sum of the o-groups $G / N_{a}(0 \neq a \in G)$. Thus $G$ is representable.

CoRollary 3.2. If $G$ is a representable 1-group, then $N\left(G_{\gamma}\right)=$ $N\left(G^{\gamma}\right)$ for each $\gamma \in \Gamma$, where $N(X)$ denotes the normalizer of $X$ in $G$. Hence $G_{\gamma}$ is normal in $G^{\gamma}$ for each $\gamma \in \Gamma$.

Proof. For $\gamma$ in $\Gamma$ and $x$ in $G, x+G^{\gamma}-x$ covers $x+G_{\gamma}-x$. Thus if $x \in N\left(G_{\gamma}\right)$ it follows that $x+G^{\gamma}-x=G^{\gamma}$. Conversely if $x \in N\left(G^{\gamma}\right), G^{\gamma}=x+G^{\gamma}-x$ covers $x+G_{\gamma}-x$. By the theorem $G_{\gamma}$ and $x+G_{\gamma}-x$ are comparable. Thus $x+G_{\gamma}-x=G_{\gamma}$.

CoRollary 3.3. Let $G$ be a representable 1-group and let $0 \neq g \in G$. Then the mapping $G_{\gamma} \rightarrow I_{\gamma}=\bigcap\left\{x+G_{\gamma}-x \mid x \in G\right\}$ is a one to one mapping of the set of all values of $g$ onto the set of all ideal values of g. Moreover, $I_{\gamma}$ is prime and is the largest 1-ideal of $G$ contained in $G_{\gamma} . \quad I^{\gamma}=\bigcup\left\{x+G^{\gamma}-x \mid x \in G\right\}$, hence $I_{\gamma} \subseteq G_{\gamma} \subset G^{\gamma} \subseteq I^{\gamma}$. Finally $G_{\gamma}$ is an essential subgroup if and only if $I_{\gamma}$ is an essential 1-ideal, and if $I_{\gamma}$ is essential, then $G_{\lambda}$ is essential for all $\lambda \in \Gamma$ such that $G_{\lambda} \supseteqq I_{\gamma}$.

Proof. The first part of this corollary follows trivially from Theorem 2.1 and 3.1. We prove only the last sentence. Suppose $G_{\gamma}$ is an essential subgroup. Then $G_{\gamma} \supseteqq R_{h} \supseteqq L_{h}$ for some $0<h$ in $G$. Since $L_{h}$ is an 1-ideal of $G, L_{h} \subseteq I_{\gamma}$. Hence $I_{\gamma}$ is an essential 1-ideal. Conversely suppose $I_{\gamma}$ is an essential 1-ideal. Then $L_{h} \cong I_{\gamma}$ for some $0<h$ in $G$. Let $I_{\beta}$ be an ideal value of $h$.

Case 1. $I_{\beta}=I_{\gamma}$. Then $h$ has only one value, namely $G_{\beta}$, and $G_{\lambda}$ is essential for all $\lambda \in \Gamma$ such that $G_{\lambda} \supseteqq G_{\beta}$. If $I_{\beta} \subseteq G_{\lambda} \subset G_{\beta}$, then 
pick $0<x \in G^{\lambda} \mid G_{\lambda}$. Since $G_{\lambda}$ is prime, $x \wedge h \in G^{\lambda} \mid G_{\lambda}$ and $x \wedge h$ has $G_{\lambda}$ for its only value. Thus $R_{x \wedge h} \subseteq G_{\lambda}$ and so $G_{\lambda}$ is essential.

Case 2. $I_{\beta} \subset I_{\gamma}$. Then $I_{\beta} \subseteq G_{\beta} \subset I^{\beta} \subseteq I_{\gamma}$. Therefore $R_{h} \subseteq I_{\gamma}$ and $G_{\lambda}$ is essential for all $\lambda \in \Gamma$ such that $G_{\lambda} \supseteqq I_{\gamma}$.

\section{CoRollary 3.4. For a representable 1-group $G, R(G)=L(G)$.}

Proof. As observed in $\S 2, L(G) \subseteq R(G)$. Let $0 \neq g \in G$. If $g$ has at least two values, then by the preceding corollary we have $I_{\alpha} \subseteq G_{\alpha} \subset L_{g}$ where $\alpha$ is a value of $g$. Thus $R(G) \subseteq R_{g} \subseteq L_{g}$. Suppose that $g$ has only one value, say $\alpha$. Then $L_{g}=I_{\alpha}$ and $I_{\alpha}$ is an essential 1-ideal. Thus $G_{\lambda}$ is essential for all $\lambda \in \Gamma$ such that $G_{\lambda} \supseteqq I_{\alpha}$ by Corollary 3.3. Moreover $L_{g}=\bigcap\left\{G_{\lambda} \mid G_{\lambda} \supseteqq I_{\alpha}\right\} \supseteqq R(G)$, as $R(G)$ is the intersection of all essential subgroups of $G$. Thus $R(G) \subseteq L_{g}$ for all $0 \neq g$ in $G$ and it follows that $R(G) \cong L(G)$.

It was pointed out in [4] that in general these radicals are not the same. Also in [4] Conrad showed that an 1-group is representable if and only if each regular 1 -ideal is prime. It is easy to construct examples to show that the converse to Corollary 3.2 and the converse to Corollary 3.4 are not true.

Proposition 3.5. (Weinberg [12]). An 1-group $G$ is completely distributive if and only if for each $0<g$ in $G$ there exists $0<g^{*}$ in $G$ such that $g=\bigvee g_{\alpha}(\alpha \in A), g_{\alpha} \in G^{+}$implies $g^{*} \leqq g_{\alpha}$ for some $\alpha \in A$.

For $g$ in $G$ let $L(g)$ denote the 1-ideal of $G$ generated by $g$. We shall call $h$ in $G t$-subordinate to $g$ if whenever $|g|=g_{1} \vee g_{2}, 0 \leqq g_{i} \in G$, then $h \in L\left(g_{i}\right)$ for $i=1$ or $i=2$. We shall use the notation $h \prec g$ to signify that $h$ is $t$-subordinate to $g$. Let $T(G)=\{g \in G \mid h \prec g$ implies $h=0\}$. In [6] Fuchs defines $h$ to be subordinate to $g$ if whenever $|g|=g_{1} \vee \cdots \vee g_{n}, 0 \leqq g_{i} \in G$ implies $h \in L\left(g_{i}\right)$ for some $i$. There he shows $\{g \in G \mid h$ is subordinate to $g$ implies $h=0\}=L(G)$. A proof of this given by a trivial modification of the proof of the next lemma. The hypothesis of representability enables us to cut $n$ down to 2 .

LEMMA 3.6. Let $G$ be a representable 1-group and let $0 \neq h \in G$. Then $h$ is not $t$-subordinate to $g$ in $G$ if and only if $g \in L_{h}$.

Proof. Suppose $h$ is not $t$-subordinate to $g$. Then there exists $0 \leqq g_{1}, g_{2}$ in $G$ such that $|g|=g_{1} \vee g_{2}$ and $h \notin L\left(g_{1}\right) \cup L\left(g_{2}\right)$. Therefore $g \in\left[L\left(g_{1}\right) \cup L\left(g_{2}\right)\right] \leqq L_{h}$. Conversely suppose $g \in L_{h}$. Let $\Delta$ be the set of ideal values of $h$. Then $L_{h}=\left[\bigcup\left\{I_{\delta} \mid \delta \in \Delta\right\}\right]$ and so $g \in L_{h}$ implies $g \in\left[\bigcup_{i=1}^{n}\left\{I_{\delta_{i}} \mid \delta_{i} \in \Delta\right\}\right]$. By Corollary 3.3 each $I_{\delta_{i}}$ is prime. Thus by Corollary 2.2, $g \in\left[I_{\delta_{1}} \cup I_{\delta_{k}}\right]$ for some $k, 1 \leqq k \leqq n$, say $k=2$. Then 
$|g|=g_{1} \vee g_{2}$ ([4], Lemma 4) where $0 \leqq g_{i} \in I_{\delta_{i}}$. Since the $I_{\delta}$ 's are ideal values of $h$ we have $h \notin I_{\delta_{1}} \cup I_{\delta_{2}} \supseteqq L\left(g_{1}\right) \cup L\left(g_{2}\right)$. Therefore $h$ is not $t$-subordinate to $g$.

Proposition 3.7. If $G$ is a representable 1-group, then $T(G)=$ $L(G)=R(G)$.

Proof. Let $g \in T(G)$. Then for each $0 \neq h$ in $G, h$ is not $t$ subordinate to $g$. Thus $g \in L_{h}$ for all $0 \neq h \in G$ and so $g \in L(G)$. Conversely if $g \in L(G)$, then $g \in L_{h}$ for all $0 \neq h$ in $G$. Therefore $h$ is not $t$-subordinate to $g$ for all $0 \neq h \in G$ and so $g \in T(G)$.

Proposition 3.8. Let $G$ be a representable 1-group. Then $G$ is completely distributive if and only if for each $0<g$ in $G$ there exists $0<g^{*}$ in $G$ such that whenever $g=g_{1} \vee g_{2}, g_{i} \in G^{+}$, then $g^{*} \leqq g_{1}$ or $g^{*} \leqq g_{i}$.

Proof. Suppose the condition is satisfied. Then for each $0<g$ in $G, g^{*}$ is $t$-subordinate to $g$. Therefore $0=T(G)=L(G)$. By the theorem in [4], $G$ is completely distributive. The converse follows trivially from the Weinberg condition stated in Proposition 3.5.

4. The Holland representation. For each $\lambda \in \Lambda$ let $T_{\lambda}$ be a totally ordered set and let $P\left(T_{\lambda}\right)$ be the o-permutation group on $T_{\lambda}$. Let $H=\Pi P\left(T_{\lambda}\right)(\lambda \in \Lambda)$ be the large cardinal product of the $P\left(T_{\lambda}\right)$ and let $\sigma_{\lambda}$ denote the projection map of $H$ onto the 1-group $P\left(T_{\lambda}\right)$. The pair $(\sigma, H)$ is an $H$-representation of an 1-group $G$ if $\sigma$ is an 1-isomorphism of $G$ into $H$ such that $G \sigma \sigma_{\lambda}$ acts transitively on $T_{\lambda}$ for all $\lambda$ in 1 . The main result of [7] is that each 1-group has an $H$-representation. A set $\left\{C_{\lambda} \mid \lambda \in \Lambda\right\}$ of prime subgroups of $G$ is an $H$-kernel if $\bigcap\left\{C_{\lambda} \mid \lambda \in \Lambda\right\}$ contains no nonzero 1-ideal of $G$. The $H$ representation $(\sigma, H)$ is called complete if $\sigma$ preserves all joins and meets that exist in $G$. In the 1-groups $P\left(T_{\lambda}\right)$ it is convenient to use multiplicative notation for the group operation since composition of function is the group operation and $f \in P\left(T_{\lambda}\right)$ is defined to be positive if $t f \geqq t$ for all $t$ in $T_{\lambda}$.

To prove a convex 1-subgroup is closed it is not difficult to show it suffices to consider only positive elements. Clearly the intersection of closed subgroups is closed.

Proposition 4.1. If $G_{\gamma}$ is an essential subgroup of an 1-group $G$, then $G_{\gamma}$ is closed.

Proof. Suppose (by way of contradiction) that there exists 
$\left\{g_{\alpha} \in G_{\gamma}^{+} \mid \alpha \in A\right\}$ such that $g=\bigvee g_{\alpha} \notin G_{\gamma}$. Let $\lambda$ be a value of $g$ such that $G_{\gamma} \subseteq G_{\lambda}$.

Case 1. There exists $0<h$ in $G$ such that $R_{h} \leqq G_{\gamma}$ and $h \in G_{\gamma}$. Then $G_{\lambda}-h+g-g_{\alpha}=G_{\lambda}+g-g_{\alpha}>G_{\lambda}$ for all $\alpha$ and $\lambda$ is a value of $-h+g-g_{\alpha}$. Let $\delta$ be any other value of $-h+g-g_{\alpha}$. Then $h \in G_{\delta}$, for otherwise $G_{\delta} \subseteq R_{h} \subseteq G_{\gamma} \subseteq G_{\lambda}$ and so $\delta=\lambda$. Thus

$$
G_{\delta}-h+g-g_{\alpha}=G_{\delta}+g-g_{\alpha}>G_{\delta} \text {. }
$$

Therefore $-h+g-g_{\alpha}>0$ ([3], p. 114) for all $\alpha$. This implies $-h+g \geqq \bigvee g_{\alpha}=g$, a contradition.

Case 2. For all $h>0$ such that $R_{h} \leqq G_{\gamma}, h \notin G_{\gamma}$. Thus $\gamma$ is the only value of $h$. Now $0 \leqq h \wedge g_{\alpha} \in G_{\gamma}$ for all $\alpha$ in $A$. Suppose $0<h \wedge g_{\alpha}$ for some $\alpha$ and let $G_{\beta}$ be a value of $h \wedge g_{\alpha}$. Then $h \notin G_{\beta}$ so $G_{\beta} \subseteq G_{\gamma}$. Since $h \wedge g_{\alpha} \in G_{\gamma}$ we have $G_{\beta} \subset G_{\gamma}$. Thus $R_{h \wedge g_{\alpha}} \subseteq G_{\gamma}$. But this is impossible by our assumption. Thus $0=h \wedge g_{\alpha}$ for all $\alpha$ in $A . \quad 0<g, h \notin G_{\gamma}$ implies $g \wedge h>0$ as $G_{\gamma}$ is prime. But then $0=\mathbf{V}\left(h \wedge g_{\alpha}\right)=h \wedge\left(\mathbf{V} g_{\alpha}\right)=h \wedge g>0$, a contradiction. This completes the proof of the proposition.

Corollary 4.2. For an 1-group $G, R(G)$ is closed.

Proof. $R(G)$ is the intersection of all essential subgroups of $G$.

COROLlaRY 4.3. If $G$ is a representable 1-group and if $I_{\nu}$ is an essential 1-ideal, then $I_{\gamma}$ is closed.

Proof. By Corollary 3.3, $G_{\lambda}$ is an essential subgroup of $G$ for all $\lambda \in \Gamma$ such that $G_{\lambda} \supseteqq I_{\gamma}$ and $I_{\gamma}=\bigcap\left\{G_{\lambda} \mid G_{\lambda} \supseteqq I_{\gamma}\right\}$.

If $L$ and $L^{\prime}$ are lattices and $\pi$ is a mapping of $L$ into $L^{\prime}$ such that $(a \vee b) \pi=a \pi \vee b \pi$ and $(a \wedge b) \pi=a \pi \wedge b \pi$ for all $a, b \in L$, then $\pi$ is called a lattice homomorphism. If, in addition, $\pi$ preserves all joins and meets that exist in $L$, then $\pi$ is said to be complete. If $\pi$ is the natural mapping of $G$ onto the lattice $r(C)$ of right cosets of $C$, where $C$ is a convex 1-subgroup of $G$, then $\pi$ is a lattice homomorphism. The following lemma was proven in [12] for 1-ideals.

Lemma 4.4. Let $C$ be a convex 1-subgroup of $G$ and let $\pi$ be the natural mapping of $G$ onto $r(C)$. Then $\pi$ is complete if and only if $C$ is closed.

Proof. Suppose $C$ is closed and let $\left\{g_{\alpha} \mid \alpha \in A\right\} \subseteq G$ such that $g=\mathrm{V} g_{\alpha}$ exists in $G$. Then $C+g \geqq C+g_{\alpha}$ for all $\alpha$. Suppose (by way of contradiction) that there exists $y$ in $G$ such that 


$$
C+g>C+y \geqq C+g_{\alpha}
$$

for all $\alpha$. Then

$$
C+g=C+g \vee C+y=C+g \vee y>C+y,
$$

so $g \vee y-y \notin C$. On the other hand $C \geqq C+g_{\alpha}-y$ so

$$
C=C+g_{\alpha}-y \vee C=C+\left(g_{\alpha}-y\right) \vee 0
$$

for all $\alpha$. Thus $\left(g_{\alpha}-y\right) \vee 0 \in C$ for all $\alpha$. Therefore

$$
(g \vee y)-y=\left(\mathbf{V} g_{\alpha}\right) \vee y-y=\left(\mathbf{V}\left(g_{\alpha}-y\right)\right) \vee 0=\mathbf{V}\left(\left(g_{\alpha}-y\right) \vee 0\right) \text {. }
$$

Since $C$ is closed, $g \vee y-y \in C$, a contradiction. The converse is trivial.

The next lemma can be proven by a direct application of Proposition 3.5 and the proof will be omitted.

Lemma 4.5. Let $H=\Pi H_{\lambda}(\lambda \in \Lambda)$ be the large cardinal product of the 1-groups $H_{\lambda}$. Then $H$ is completely distributive if and only if $H_{\lambda}$ is completely distributive for all $\lambda \in \Lambda$.

THEOREM 4.6. For an 1-group $G$, the following are equivalent.

(1) $G$ has a complete $H$-representation.

(2) $G$ has an H-kernel $\left\{C_{\lambda} \mid \lambda \in \Lambda\right\}$ where each $C_{\lambda}$ is closed.

Proof. (1) implies (2). Suppose $(\sigma, H)$ is a complete $H$-representation of $G$, where $H, \sigma$ and $\sigma_{\lambda}$ are as in the beginning of this section. For each $\lambda \in A$ pick $t_{\lambda} \in T_{\lambda}$ and let $C_{\lambda}=\left\{g \in G \mid t_{\lambda} g \sigma \sigma_{\lambda}=t_{\lambda}\right\}$. Then $C_{\lambda}$ is a prime subgroup (see [7], Theorem 3). Suppose $0<h \in \bigcap\left\{C_{\lambda} \mid \lambda \in \Lambda\right\}$. Then $h \sigma \sigma_{\lambda}>\theta_{\lambda}$ for some $\lambda$, where $\theta_{\lambda}$ denotes the identity in $P\left(T_{\lambda}\right)$. Then there exists $s$ in $T_{\lambda}$ with $s \neq t_{\lambda}$ and $s<s h \sigma \sigma_{\lambda}$. Since $G \sigma \sigma_{\lambda}$ acts transitively on $T_{\lambda}$, there exists $g$ in $G$ such that $t_{\lambda} g \sigma \sigma_{\lambda}=s$. Therefore $t_{\lambda}(g+h-g) \sigma \sigma_{\lambda} \neq t_{\lambda}$. Thus $\bigcap\left\{C_{\lambda} \mid \lambda \in \Lambda\right\}$ contains no nonzero 1-ideal of $G$ and hence $\left\{C_{\lambda} \mid \lambda \in \Lambda\right\}$ is an $H$-kernel. Since polars are closed the projection map of $H$ onto a cardinal summand is complete. Suppose $\left\{g_{\alpha} \mid \alpha \in A\right\} \subseteq C_{\lambda}$ such that $\mathrm{V} g_{\alpha}$ exists. Then

$$
t_{\lambda}\left(\mathrm{V} g_{\alpha}\right) \sigma \sigma_{\lambda}=t_{\lambda}\left(\mathbf{V}\left(g_{\alpha} \sigma \sigma_{\lambda}\right)\right)=t_{\lambda}
$$

by a theorem of J. T. Lloyd ([8], Theorem 1.3). Therefore $\mathrm{V} g_{\alpha} \in C_{\lambda}$ and hence each $C_{\lambda}$ is closed.

(2) implies (1). Let $\left\{C_{\lambda} \mid \lambda \in \Lambda\right\}$ be as in (2). For each $\lambda \in \Lambda$ let $P\left(r\left(C_{\lambda}\right)\right)$ be the o-permutation group on the totally ordered set $r\left(C_{\lambda}\right)$ of right cosets of $C_{\lambda}$. For $g$ in $G$ and $\lambda$ in $\Lambda$ we define a mapping $\sigma_{\lambda}$ from $G$ into $P\left(r\left(C_{\lambda}\right)\right)$ by $\left(C_{\lambda}+x\right) g \sigma_{\lambda}=C_{\lambda}+x+g$. It is easy to 
verify (or see [7]) that $\sigma_{\lambda}$ is an 1-homomorphism of $G$ onto a transitive 1-subgroup of $P\left(r\left(C_{\lambda}\right)\right)$. Let $H=\Pi P\left(r\left(C_{\lambda}\right)\right)(\lambda \in \Lambda)$ be the large cardinal product of the 1-groups $P\left(r\left(C_{\lambda}\right)\right)$. We define a mapping $\sigma$ of $G$ into $H$ by $g \sigma=\left(\cdots, g \sigma_{\lambda}, \cdots\right)$. Then $\sigma$ is an 1-homomorphism of $G$ into $H$ and the kernel of $\sigma$,

$$
K(\sigma)=\left\{g \in G \mid x+g-x \in C_{\lambda} \text { for all } x \in G, \lambda \in \Lambda\right\} \subseteq \bigcap C_{\lambda}(\lambda \in \Lambda) .
$$

Since this intersection contains no nonzero 1-ideals, $\sigma$ is an 1-isomorphism. Therefore $(\sigma, H)$ is an $H$-representation of $G$. Let $\left\{g_{\alpha} \mid \alpha \in A\right\} \subseteq G$ such that $\mathbf{V} g_{\alpha}$ exists. Since the $C_{\lambda}$ 's are closed we have by Lemma 4.4 that for each $\lambda$ in $\Lambda$,

$$
\begin{gathered}
\left(C_{\lambda}+x\right)\left(\mathbf{V} g_{\alpha}\right) \sigma_{\lambda}=C_{\lambda}+x+\mathbf{V} g_{\alpha}=C_{\lambda}+\mathbf{V}\left(x+g_{a}\right) \\
=\mathrm{V}\left(C_{\lambda}+x+g_{\alpha}\right)=\mathrm{V}\left(\left(C_{\lambda}+x\right) g_{\alpha} \sigma_{\lambda}\right) .
\end{gathered}
$$

For $h$ in $H$ the following are equivalent. $h \geqq g_{\alpha} \sigma$ for all $\alpha$; $(h)_{\lambda} \geqq g_{\alpha} \sigma_{\lambda}$ for all $\alpha$ and all $\lambda ;(h)_{\lambda} \geqq \bigvee\left(g_{\alpha} \sigma_{\lambda}\right)=\left(\mathbf{V} g_{\alpha}\right) \sigma_{\lambda}$ for all $\lambda ; h \geqq\left(\mathbf{V} g_{\alpha}\right) \sigma$. Therefore $\sigma$ is complete. This concludes the proof of the theorem.

COROLlary 4.7. If G satisfies (1) and (2) of the theorem, then $G$ is completely distributive.

Proof. J. T. Lloyd has proven ([8], Theorem 1.1) that for an ordered set $T, P(T)$ is completely distributive. Thus by Lemma 4.5, $H$ (as above) is completely distributive. Since the $H$-representation is complete, joins and meets in $G$ "agree" (i.e., under 1-isomorphism) with those in $H$. Thus $G$ is completely distributive.

Corollary 4.8. $R(G)=0$ implies (2) of the theorem. Thus $R(G)=0$ implies $G$ is completely distributive.

Proof. $\quad R(G)=\bigcap\left\{G_{\gamma} \mid G_{\gamma}\right.$ is an essential subgroup of $\left.G\right\}$. By Proposition 4.1, each essential subgroup of $G$ is closed and an essential subgroup, being regular, is prime. Thus $\left\{G_{\gamma} \mid G_{\gamma}\right.$ is an essential subgroup of $G\}$ is an $H$-kernel as $R(G)=0$, all of whose members are closed.

In [4] Conrad observed that $R(G)=0$ implies $G$ is completely distributive and gives an example to show that the converse is false. Also it is shown in [4] that for representable 1-groups the converse to Corollary 4.7 is true. The answer to this question is not known for arbitrary 1-groups. Finally, Corollary 4.8 shows that the $H$ representation used in [9] (Theorem 2.1) is complete, as the possession of a basis for an 1-group $G$ implies $R(G)=0$. 


\section{REFERENCES}

1. S. J. Bernau, Unique representation of Archimedean lattice groups and normal Archimedean lattice rings, Proc. London Math. Soc. 15 (1965), 599-631.

2. G. Birkhoff, Lattice Theory, rev. ed., Amer. Math. Soc. Colloquim Pub. 25, 1948.

3. P. Conrad, The lattice of all convex 1-subgroups of a lattice-ordered group, Czech. Math. J. 15 (1965), 101-123.

4. - The relationship between the radical of a lattice-ordered group and complete distributivity, Pacific J. Math. 14 (1964), 494-499.

5. L. Fuchs, Partially Ordered Algebraic Systems, Pergamon Press, 1963.

6. - Riesz Groups, (to appear).

7. C. Holland, The lattice-ordered group of automorphisms of an ordered set, Michigan Math. J. 10 (1963), 399-408.

8. J. T. Lloyd, Lattice-ordered groups and o-permutation groups, Tulane Dissertation, 1964.

9. - Representations of lattice-ordered groups having a basis, Pacific J. Math. 15 (1965), 1313-1317.

10. F. Sik, Sous-groupes simples et ideaux simples des groupes réticulés, C. R. Acad. Sc. Paris 261 (1965), 2791-2793.

11. — Uber Subdirekte Summen geordneter Gruppen, Czech. Math. J. 10 (1960), 400-424.

12. E. C. Weinberg, Completely distributive lattice-ordered groups, Pacific J. Math. 12 (1962), 1131-1137.

Received May 2, 1966. This work was supported in part by National Science Foundation Grants GP1791 and 64239 and represents a portion of the author's doctoral dissertation, written under the direction of Professor Paul F. Conrad, to whom the author expresses his gratitude.

TUlane University of LOUISIANA 


\title{
PACIFIC JOURNAL OF MATHEMATICS
}

\author{
EDITORS
}

H. Samelson

Stanford University

Stanford, California

J. P. JANS

University of Washington

Seattle, Washington 98105

\section{J. DugundJI}

University of Southern California Los Angeles, California 90007

RICHARD ARENS

University of California

Los Angeles, California 90024

\section{ASSOCIATE EDITORS}

E. F. BECKENBACH

B. H. NeUmanN

F. WOLF

K. YosIDA

\section{SUPPORTING INSTITUTIONS}

UNIVERSITY OF BRITISH COLUMBIA

CALIFORNIA INSTITUTE OF TECHNOLOGY

UNIVERSITY OF CALIFORNIA

MONTANA STATE UNIVERSITY

UNIVERSITY OF NEVADA

NEW MEXICO STATE UNIVERSITY

OREGON STATE UNIVERSITY

UNIVERSITY OF OREGON

OSAKA UNIVERSITY

UNIVERSITY OF SOUTHERN CALIFORNIA

\author{
STANFORD UNIVERSITY \\ UNIVERSITY OF TOKYO \\ UNIVERSITY OF UTAH \\ WASHINGTON STATE UNIVERSITY \\ UNIVERSITY OF WASHINGTON \\ * \\ AMERICAN MATHEMATICAL SOCIETY \\ CHEVRON RESEARCH CORPORATION \\ TRW SYSTEMS \\ NAVAL ORDNANCE TEST STATION
}

Mathematical papers intended for publication in the Pacific Journal of Mathematics should be typewritten (double spaced). The first paragraph or two must be capable of being used separately as a synopsis of the entire paper. It should not contain references to the bibliography. Manuscripts may be sent to any one of the four editors. All other communications to the editors should be addressed to the managing editor, Richard Arens at the University of California, Los Angeles, California 90024.

50 reprints per author of each article are furnished free of charge; additional copies may be obtained at cost in multiples of 50 .

The Pacific Journal of Mathematics is published monthly. Effective with Volume 16 the price per volume (3 numbers) is $\$ 8.00$; single issues, $\$ 3.00$. Special price for current issues to individual faculty members of supporting institutions and to individual members of the American Mathematical Society: $\$ 4.00$ per volume; single issues $\$ 1.50$. Back numbers are available.

Subscriptions, orders for back numbers, and changes of address should be sent to Pacific Journal of Mathematics, 103 Highland Boulevard, Berkeley 8, California.

Printed at Kokusai Bunken Insatsusha (International Academic Printing Co., Ltd.), No. 6, 2-chome, Fujimi-cho, Chiyoda-ku, Tokyo, Japan.

PUBLISHED BY PACIFIC JOURNAL OF MATHEMATICS, A NON-PROFIT CORPORATION

The Supporting Institutions listed above contribute to the cost of publication of this Journal, but they are not owners or publishers and have no responsibility for its content or policies. 


\section{Pacific Journal of Mathematics}

\section{Vol. 20, No. $3 \quad$ November, 1967}

Dallas O. Banks, Lower bounds for the eigenvalues of a vibrating string whose density satisfies a Lipschitz condition ................... 393

Ralph Joseph Bean, Decompositions of $E^{3}$ which yield $E^{3} \ldots \ldots \ldots \ldots 411$

Robert Bruce Brown, On generalized Cayley-Dickson algebras ......... 415

Richard Dowell Byrd, Complete distributivity in lattice-ordered groups . . . 423

Roger Countryman, On the characterization of compact Hausdorff $X$ for

which $C(X)$ is algebraically closed ...................... 433

Cecil Craig, Jr. and A. J. Macintyre, Inequalities for functions regular and bounded in a circle ...................................... 449

Takesi Isiwata, Mappings and spaces ..................... 455

David Lewis Outcalt, Power-associative algebras in which every subalgebra is an ideal ..................................... 481

Sidney Charles Port, Equilibrium systems of stable processes .......... 487

Jack Segal, Quasi dimension type. I. Types in the real line ............ 501

Robert William Stringall, Endomorphism rings of primary abelian

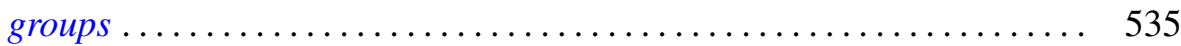

William John Sweeney, "The $\delta$-Poincaré estimate” ................. 559

L. Tzafriri, Operators commuting with Boolean algebras of projections of finite multiplicity ............................... 571 